\title{
Tensile properties of 3D multi-layer wrapping braided composite: Progressive damage analysis
}

\author{
Xiaopei Wang ${ }^{\mathrm{a}, \mathrm{b}}$, Deng'an Cai ${ }^{\mathrm{a}}$, Vadim V. Silberschmidt ${ }^{\mathrm{b}}$, Jian Deng ${ }^{\mathrm{a}}$, Honglei Tian ${ }^{\mathrm{c}}$, \\ Guangming Zhou ${ }^{\text {a," }}$ \\ ${ }^{a}$ State Key Laboratory of Mechanics and Control of Mechanical Structures, Nanjing University of Aeronautics and Astronautics, Nanjing, 210016, PR China \\ ${ }^{\mathrm{b}}$ Wolfson School of Mechanical, Electrical and Manufacturing Engineering, Loughborough University, Leicestershire, LE11 3TU, UK \\ c CRRC, Qingdao Sifang Rolling Stock Research Institute Co Ltd, Qingdao, 266111, PR China
}

\begin{abstract}
An experimental and numerical study on mechanical properties and damage behavior of 3D multi-layer wrapping braided composite under axial tensile load is presented. The braiding process of this material is introduced and its tensile properties are obtained in tensile tests. Numerical simulations employ periodical boundary conditions, with interface elements between yarns and matrix added to improve the accuracy of prediction. 3D Hashin-type criteria and Von-Mises stress criterion are employed as damage initiation criteria for yarns and matrix, respectively. The obtained numerical results show a good agreement with the experimental data. The load-bearing capacity and failure mechanisms of 3D multi-layer wrapping braided composites under axial tensile loading are also discussed. A stress distribution shows that the axial yarns are the main load-bearing component of the composite. The main failure mode of the yarns is the yarn-matrix tensile cracking in the width direction, followed by the yarn-matrix tensile cracking in the thickness direction and fibre tensile failure. When the fibres in axial yarns begin to break, the material loses its load-bearing capacity.
\end{abstract}

\author{
Keywords: \\ 3D braided composite \\ Multi-layer wrapping \\ Progressive damage analysis \\ Mechanical properties \\ Damage mechanism
}

\section{Introduction}

Three-dimensional (3D) braided composites are widely used due to their lightweight, high stiffness- and strength-to-weight ratios, good fatigue and corrosion resistance. Compared with traditional laminated composites, 3D braided composites have the advantages of higher structural integrity, better impact damage resistance and higher through-thickness properties [1-4].

For 3D braided composites, there are many theoretical and numerical studies predicting elastic properties and assessing the effect of micro structure [5-13]. Kalidinidi et al. [5] revealed that the weighted averaged isostrain and isostress models yielded the best comparisons against the experiments. Sankar et al. [6] proposed a selective averaging method for prediction of elastic parameters of textile composite materials. A model known as the binary model was formulated by Cox et al. $[7,8]$ to describe the behavior of woven composites with 3D interlock reinforcement. Tang et al. [10] analyzed a geometric model and assumed the existence of different types of unit cells in three regions of the 3D braided composite - interior, surface and corner. On the basis of these types of unit cells and the variational principle, a finite-multiphase-element method was proposed to predict the effective elastic properties of 3D four directional braided composites by Chen et al. [11]. Sun et al. [12] used the volume averaging method to calculate the total stiffness and engineering elastic constants of 3D surface-core four-directional braided composites and discussed the effect of a braiding angle and a fibre volume fraction on their stiffness. Li et al. [13] established a parameterized finite-element model of 3D five-directional rectangular braded composites, which precisely simulated a spatial configuration of braiding yarns and considered cross-section deformation as well as a surface contact relationship between the yarns.

In recent years, the research on strength characteristics of 3D braided composites developed gradually [14-20]. Sun et al. [14] proposed a fibre-inclination model to predict strength of 3D braided composites, based on transverse isotropy of unidirectional laminae and the Tsai-Wu polynomial failure criterion. Zuo et al. [15] calculated the statistical tensile strength of 3D four-directional braided composites with an approach based on a statistical model for tensile strength of

\footnotetext{
* Corresponding author. Address: No.29 Yudao Street, Nanjing University of Aeronautics and Astronautics, Nanjing, 210016, PR China.

E-mail address: zhougm@nuaa.edu.cn (G. Zhou).
} 
unidirectional composite materials. Yu et al. [16] applied a two-scale method to predict the effect of braiding angle and fibre volume fraction on tensile, bending and torsion strengths of 3D braided composites. A maximum-normal-stress criterion was used as a longitudinal-strength criterion for fibres, while a Von-Mises effective-stress yield criterion was employed for matrix. Based on a 3D 5-directional braiding process, $\mathrm{Li}$ et al. [17] established three types of microstructural unit-cell models, respectively for the interior, surface and corner regions, and employed a Hoffman criterion to detect the tensile failure of fibre bundles. Jiang et al. [18] presented a theoretical model based on a helix geometry unit cell with prediction of effective elastic constants and failure strength of 3D braided composites under uniaxial load with a volume-average stiffness method and the Tsai-Wu polynomial failure criterion. Wang et al. [19] predicted tensile strength and progressive damage behavior of braided textile composites with a multi-scale modelling approach, with 3D Hashin and Stassi failure criteria employed with a modified Murakami-type stiffness-degradation scheme. Besides several damage modes of braiding yarns and matrix of 3D 4-directional braided composites, Fang et al. [20] considered an interface damage mode using a cohesive-zone model in numerical simulations. An influence of interface properties on a macroscopic stress-strain curve and interaction of different failure modes of the braided composites under uniaxial tensile loading were evaluated.

Compared with analysis of stiffness, prediction of strength characteristics for 3D braided composites is more difficult and less accurate because of their complex mesoscopic structures. Additionality, the complex micro-structure also leads to the problems of high cost, impermeability or high porosity of 3D braided composites in forming process. 3D multi-layer wrapping braided composites studied in this paper add radial yarns in the basis of laminates, which not only improve their interlaminar performance but are also easy to form. Deng et al. [21] studied elastic properties of this material under tension and compression loads.

In this paper, tensile properties and progressive damage of a 3D multi-layer wrapping braided composite are studied. Its braiding process is described in detail, followed by development of a representative volume cell (RVC) model with interface elements between yarns and matrix based on the braiding process. To improve the accuracy of prediction, periodical boundary conditions are introduced. 3D Hashin criteria and Von-Mises stress criterion are employed for damageinitiation criteria of yarns and matrix, respectively. To verify numerical simulations with the developed model, quasi-static tensile tests are carried out.

\section{Braiding process}

The studied 3D multi-layer wrapping braided composite contains four kinds of yarns: braiding, axial, radial and weft yarns. It was braided with a four-step braiding process. Carrier motion rules of the 3D multilayer wrapping braiding process are shown in Fig. 1. The braiding yarns moved only in the circumferential direction and directions of the adjacent rings were opposite (Fig. 1a). In order to enhance the mechanical properties in the vertical direction, the axial yarns were added between the adjacent braiding yarns. The interlaminar bonding property was strengthened by adding radial yarns at the second and the third steps. The radial yarns were only moved in-plane and successively wound around each column of braiding yarns. The only purpose of presence of weft yarns in the outer rings was to fix the radial yarns.

As seen in the unit-cell model (Fig. 1b), the radial yarn was inserted from the inner layer to outer layer in the second step. Then it bypassed the weft yarn and was inserted back to the inner layer in the third step. At the next stage, the radial yarn only rose a certain distance in the inner layer.

A representative surface of the 3D multi-layer wrapping braided preform is shown in Fig. 2, with braiding and weft yarns are seen clearly, while radial yarns appearing intermittently.

To study mechanical properties of the $3 \mathrm{D}$ multi-layer wrapping

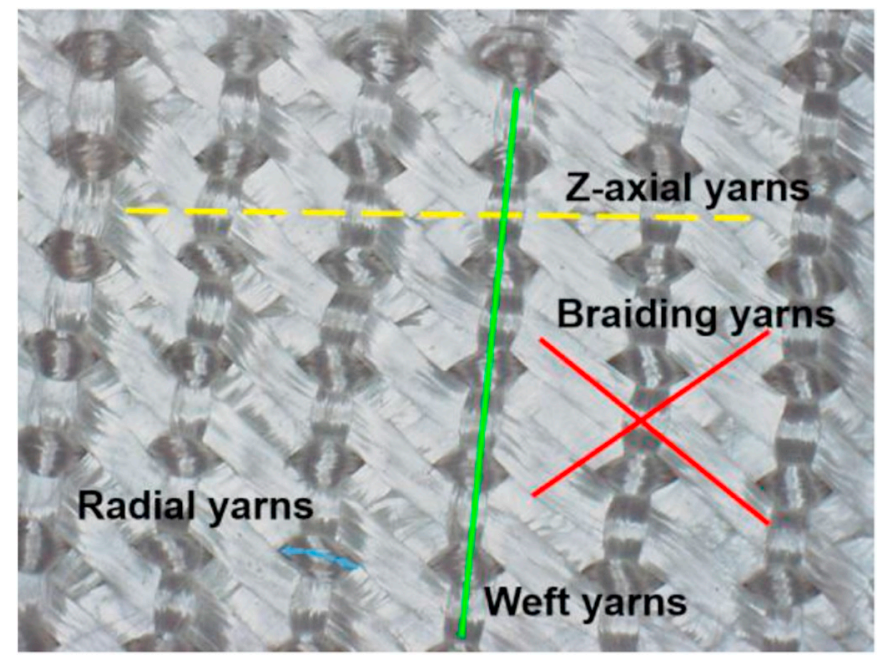

Fig. 2. Surface of 3D multi-layer wrapping braided preform.

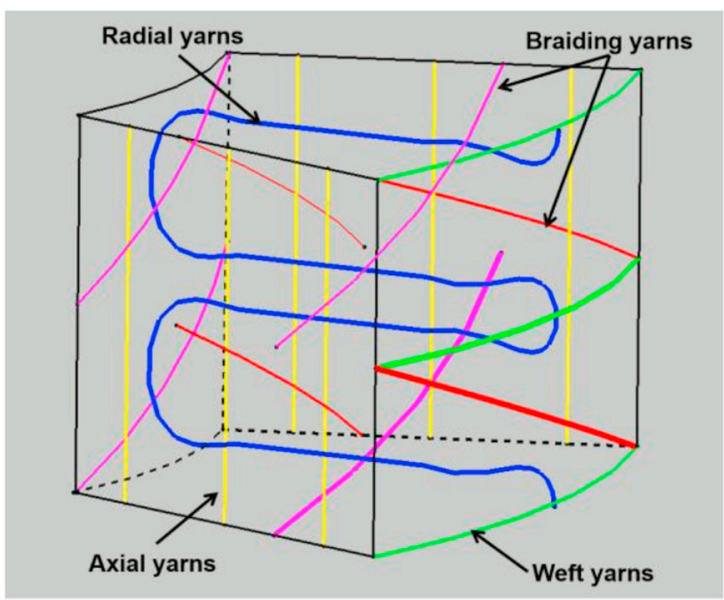

(b) (a)

Radial yarns

Braiding yarns

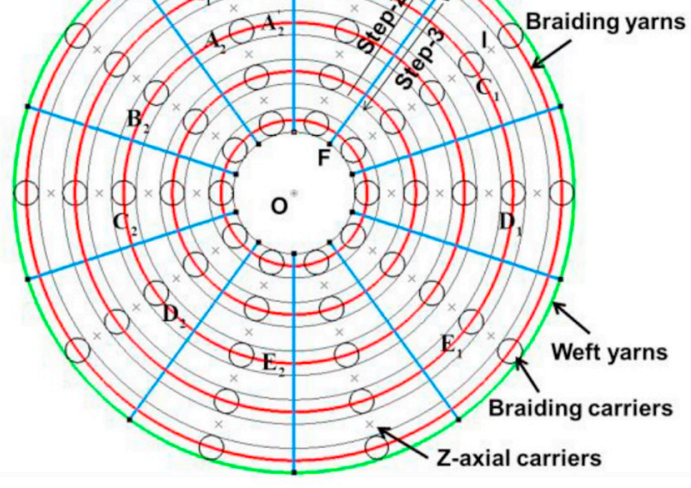

Fig. 1. 3D multi-layer wrapping braided composites: (a) carrier motion rules; (b) geometrical model of unit cell. 
braided composites, some assumptions were made in their model. Although such composites are braided with a circular braiding technology, the thickness of a tube wall is smaller than its diameter, which means that the tube can be regarded as a thin-walled structure. So, the effect of curvature of the tube wall can be ignored when considering the unit. When plate tests were carried out, the braided preform was cut along its axial direction. So, a cross section of the braided preform changed from a circular shape to a rectangle shape. Since the weft yarns have little effect on overall tensile properties, the effect of surface cells can be ignored. So, when modelling and analyzing the properties of the 3D multi-layer wrapping braided composites, only one interior cell was taken as the RVC. As many kinds of yarns were used in the 3D multilayer wrapping braided composites, the yarns were extruded and bent in space, with both the shape and dimensions of their cross sections changed after straightening. However, the effect due to these changes was relatively small for the whole model. So, in the solid structure model, the yarns were kept straight and their cross-section area and shape remained the same. Observation confirmed that the braiding and axial yarns had flat cross sections. In order to simplify the model, the cross sections of braiding and axial yarns were assumed to be rectangular, while those of radial yarns elliptical, as shown in Fig. 3.

\section{Experimental study}

\subsection{Materials and specimens}

The 3D multi-layer wrapping braided composite performs (glass fibre) were produced by Nanjing Fiberglass Research \& Design Institute, Sinoma, China. The specimens were manufactured by resin transfer molding (RTM) in the State Key Laboratory of Mechanics and Control of Mechanical Structures at Nanjing University of Aeronautics and Astronautics (China). The epoxy resin used in composite molding was Epikote E51-618; m-phenylenediamine (MPD) was used as hardener.

By measuring the height and width of the preform, the braiding angle was calculated as 45 . The fibre volume fraction was obtained by

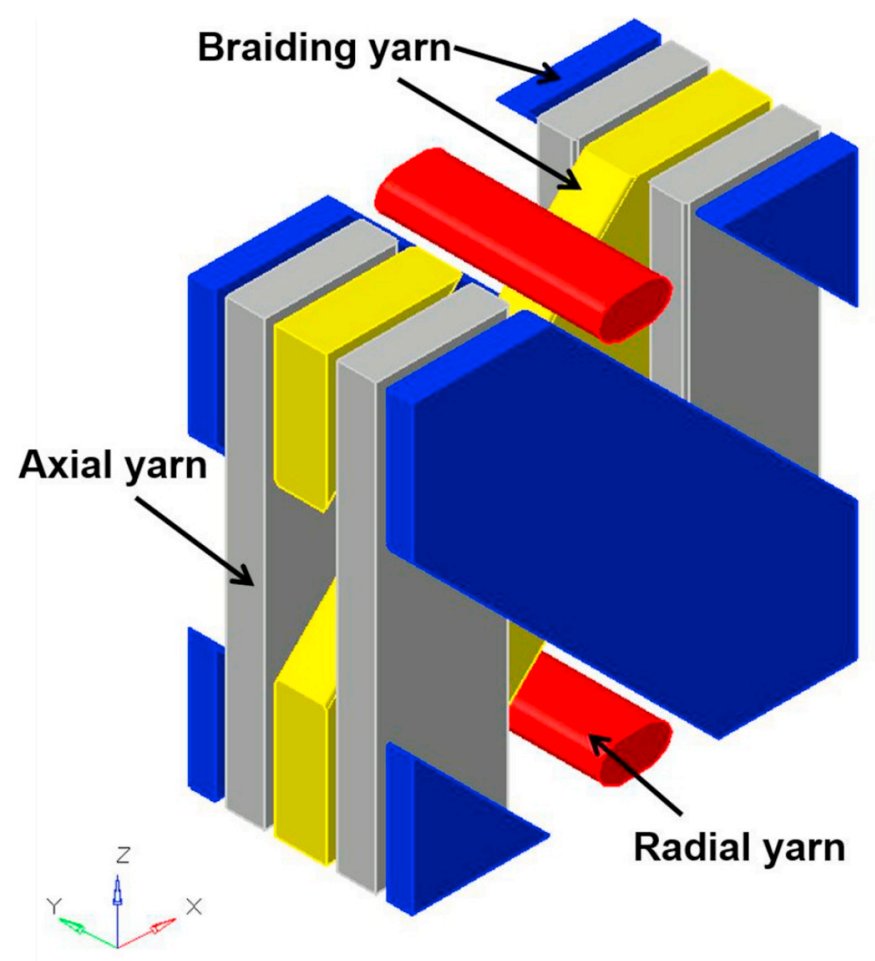

Fig. 3. Solid structure model of unit cell of 3D multi-layer wrapping braided composite. weighting the preform and the panel. It was $39.01 \%$ in this composite panel. The parameters of preforms are listed in Table 1.

The panel was cut into six specimens, each with the following dimensions: length of about $230 \mathrm{~mm}$, width of $25 \mathrm{~mm}$ and thickness of $2.25 \mathrm{~mm}$. In order to reinforce the specimen, $1 \mathrm{~mm}$-thick and $50 \mathrm{~mm}$ long aluminum plates were attached on both sides of the specimen's ends by $A B$ glue and two strain rosettes were bonded on the surface at the centre of the specimens, as shown in Fig. 4.

\subsection{Test procedure}

The quasi-static tensile tests were conducted on a MTS 370.25 test machine with a constant speed of $1 \mathrm{~mm} / \mathrm{min}$. The axial displacement and tensile force $F$ were recorded by MTS data collecting software and strains $\varepsilon_{x}$ and $\varepsilon_{y}$ were measured with the DH3816 static strain-testing system. The tensile modulus in the $x$ direction and the Poisson's ratio $v_{x y}$ were calculated with the following equations:

$$
\begin{gathered}
E_{x} \frac{\Delta \sigma_{x}}{\Delta \varepsilon_{x}}, \\
v_{x y} \frac{\Delta \varepsilon_{y}}{\Delta \varepsilon_{x}}
\end{gathered}
$$

\subsection{Experimental results}

Failure modes of the 3D multi-layer wrapping braided composite under tensile load are shown in Fig. 5. The damage locations of all the specimens in Fig. 5 were in the middle area, a part of specimen DC-L1 with failure at the root. So, the results for specimen DC-L1 were ignored. As the load increased, some noise was heard occasionally due to damage in fibres and matrix. Delamination phenomenon could not be found in the failed specimens, indicating superior delamination resistance of the 3D multi-layer wrapping braided composite.

According to Fig. 5, fibre breakage was the dominant cause of the final failure; still none of the specimens was completely separated into two parts. This indicates that some yarns were not broken when the ultimate load was reached. On both sides of the fracture surface, the matrix whitened, indicating that the matrix in these areas was damaged. The fracture surface of the studied composite was not a flat cross section perpendicular to the axial direction. On the contrary, it had a V-shape or a slope because of the braiding process. In the specimen subjected to a tensile load, it was mainly carried by the axial yarns, and their loading direction was the longitudinal one. The braiding yarns were subjected to a shear load; it means that the tensile load acted in both the transverse and longitudinal directions. The multiplicity of yarns led to complexity of a stress transfer and crack propagation; as a result, the fracture surfaces were not consistent.

The obtained tensile properties of the studied composite are presented in Table 2; the tensile modulus and the Poisson's ratio were calculated at a strain range of $0.1 \%-0.4 \%$. Repeatability and consistency of five specimens were very good. The average tensile modulus and Poisson's ratio of the 3D multi-layer wrapping braided composite were $21.7 \mathrm{GPa}$ and 0.348 , respectively. The difference between the magnitude of the tensile modulus of specimens and their average value was less than $3 \%$. The average tensile strength of the composite was $320 \mathrm{MPa}$, with the error between the maximum tensile strength and the average of $5.21 \%$, while that between the minimum tensile strength and the average $2.64 \%$.

Table 1

Parameters of preforms.

\begin{tabular}{lllll}
\hline $\begin{array}{l}\text { Height of } 10 \\
\text { unit cells } \\
(\mathrm{mm})\end{array}$ & $\begin{array}{l}\text { Width of } 10 \\
\text { unit cells } \\
(\mathrm{mm})\end{array}$ & $\begin{array}{l}\text { Weight of } \\
\text { preform }(\mathrm{g})\end{array}$ & $\begin{array}{l}\text { Weight of } \\
\text { panel }(\mathrm{g})\end{array}$ & $\begin{array}{l}\text { Fibre volume } \\
\text { fraction (\%) }\end{array}$ \\
\hline 15.0 & 15.0 & 135 & 238 & 39.01 \\
\hline
\end{tabular}




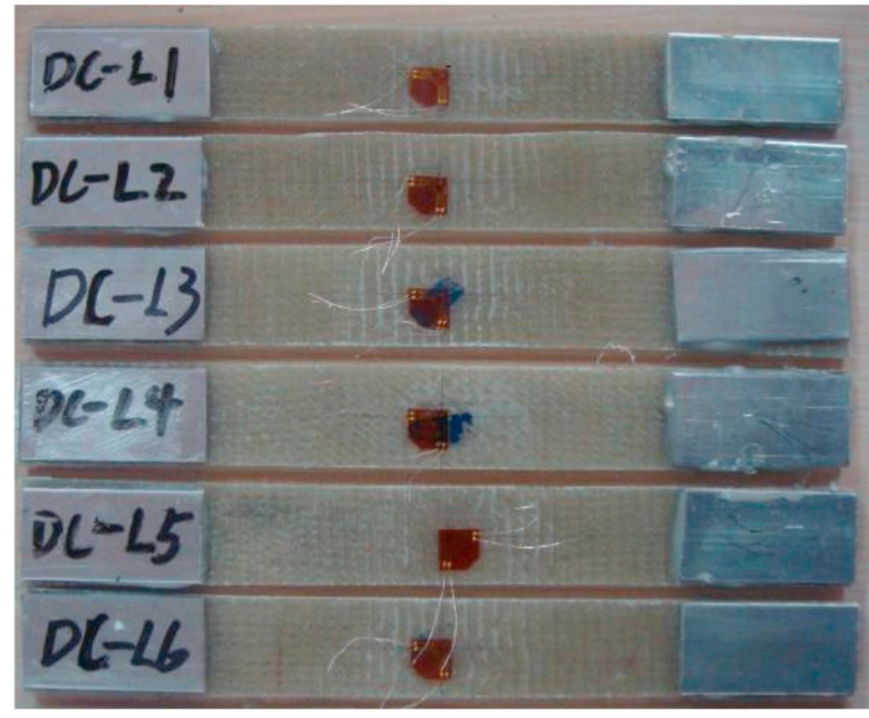

Fig. 4. Specimens of 3D multi-layer wrapping braided composites.

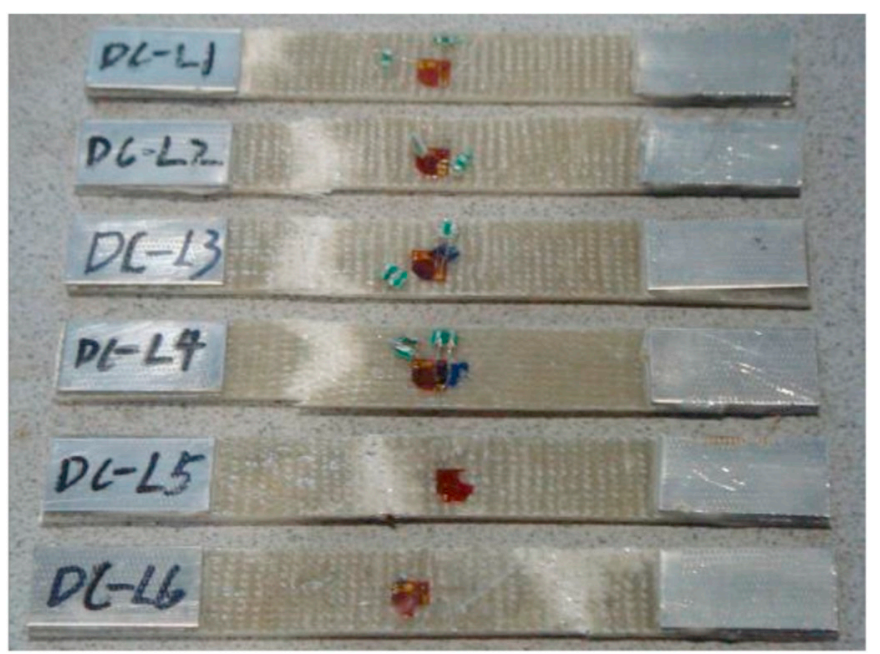

Fig. 5. Failure modes of 3D multi-layer wrapping braided composites under tensile load.

Table 2

Tensile properties.

\begin{tabular}{llll}
\hline Specimen & Modulus/GPa & Poisson's ratio $v_{x y}$ & Strength/MPa \\
\hline DC-L2 & 22.1 & 0.33 & 337 \\
DC-L3 & 21.4 & 0.34 & 315 \\
DC-L4 & 22.0 & 0.38 & 311 \\
DC-L5 & 22.1 & 0.35 & 314 \\
DC-L6 & 21.1 & 0.34 & 322 \\
\hline
\end{tabular}

\section{Finite-element analysis}

\subsection{Representative volume cell}

Based on the solid structure model in Fig. 3 and the braiding parameters from the test, the size of RVC and the cross section area of each yarn were determined. The length, width and height of RVC were $1.5 \mathrm{~mm}, 1.5 \mathrm{~mm}$ and $0.75 \mathrm{~mm}$, respectively. The cross section area of braiding yarn and axial yarn were $0.12 \mathrm{~mm}^{2}$ both, while that of radial yarn was $0.03 \mathrm{~mm}^{2}$. In order to simulate and predict behavior of the 3D multi-layer wrapping braided composite more accurately, interface cohesive elements were introduced between the yarns and the matrix, as shown in Fig. 6. Thickness of cohesive elements was $0.005 \mathrm{~mm}$. The yarns and the matrix were meshed with two types of elements: 4-node linear tetrahedron elements (C3D4) and 8-node linear brick, reducedintegration elements (C3D8R) with hourglass control, respectively. The interface was meshed with 8-node 3D cohesive elements (COH3D8).

\subsection{Periodic boundary conditions}

Numerical simulations of the studied composite were conducted on the basis of RVC. Because of the material's periodicity, the levels of displacements and stresses on the opposite surfaces of RVCs should be continuous. So, the periodical boundary conditions were applied to the RVC model. The unified periodical boundary conditions for a parallelepiped RVC proposed by Xia et al. [22] can be represented as

$\begin{array}{lll}u_{i} & \varepsilon_{i k} x_{k} & u_{i}\end{array}$

where $\varepsilon_{i k}$ are the average strains and $x_{k}$ are the Cartesian coordinates, $u_{i}$ is the periodic part of displacement, which is unknown generally. Hence, the displacements on the boundary surfaces can be expressed as

$u_{i}^{j} \quad \varepsilon_{i k} x_{k}^{j} \quad u_{i}$

$u_{i}^{j} \quad \varepsilon_{i k} x_{k}^{j} \quad u_{i}$

where $j$ and $j$-represent the positive and the negative directions along $X_{j}$, respectively. The difference between $u_{i}^{j}$ and $u_{i}^{j}$ is

$u_{i}^{j} \quad u_{i}^{j} \quad \varepsilon_{i k} \Delta x_{k}^{j}$

where $\Delta x_{k}^{j}$ is the difference between Cartesian coordinates of corresponding position. For any parallelepiped RVC model like Fig. $6, \Delta x_{k}^{j}$ are constant.

\subsection{Materials and properties}

The 3D multi-layer wrapping braided composite consisted of yarns and resin matrix. The yarns, containing E-glass fibres and the matrix, were regarded as unidirectional composites and assumed to be transversely isotropic in the local coordinate system. The resin matrix is

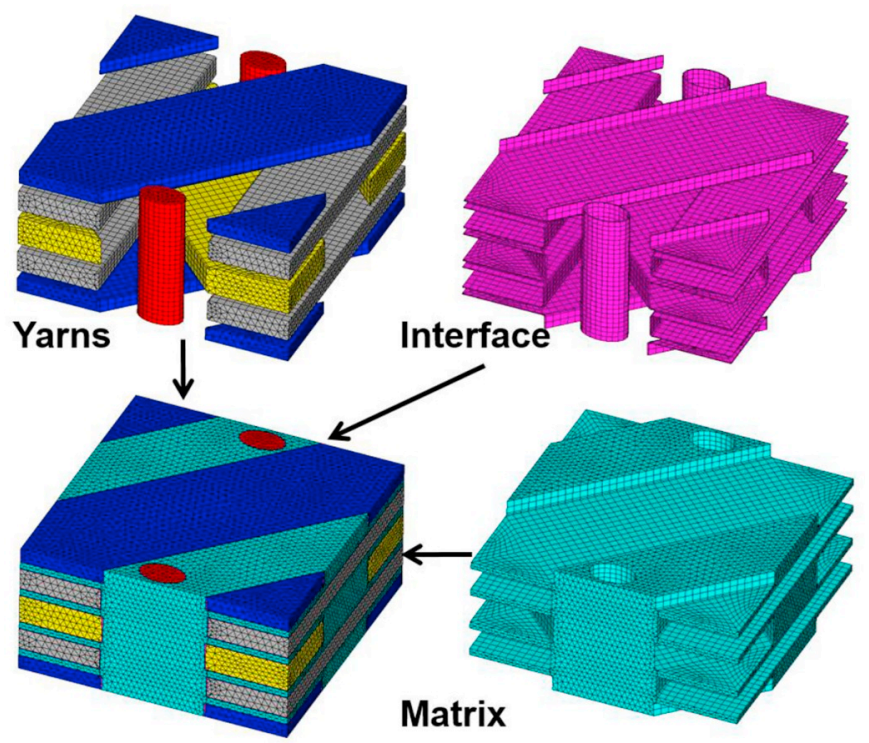

Fig. 6. Mesh generation for 3D multi-layer wrapping braided composites under tensile load. 
isotropic. Mechanical properties of the yarns were calculated employing micromechanics formulae proposed by Chamis [23]. The used material properties of yarns, matrix and their interface are listed in Tables 3 and 4. Yarn properties in Table 3 were calculated for the volume fraction of fibres of 0.8 .

\subsection{Progressive damage model}

Analyzing progressive damage, in order to reveal a failure mechanism of the 3D multi-layer wrapping braided composite and predict its strength, it is important to simulate initiation and evolution of damage in yarns. Since they are regarded as unidirectional composite with transverse isotropic properties, the 3D Hashin criteria based on literature $[24,25]$ can be employed as damage initiation criteria. The criteria are formulated as follows:

Fibre tensile failure (SDV1), for $\sigma_{11} \quad 0$ :

$\varnothing_{1 T} \quad \frac{\sigma_{11}}{X_{1 T}}{ }^{2}\left(\begin{array}{ll}\frac{\tau_{12}^{2}}{2 G_{12}} & \frac{3}{4} \alpha_{s} \tau_{12}^{4} \\ \frac{S_{12}^{2}}{2 G_{12}} & \frac{3}{4} \alpha_{S} S_{12}^{4}\end{array}\right)^{2} \quad\left(\begin{array}{cc}\frac{\tau_{13}^{2}}{2 G_{13}} & \frac{3}{4} \alpha_{s} \tau_{13}^{4} \\ \frac{S_{13}^{2}}{2 G_{13}} & \frac{3}{4} \alpha_{s} S_{13}^{4}\end{array}\right)^{2} 1$

Fibre compressive failure and fibre-matrix shear-out (SDV2), for $\sigma_{11}<0$ :

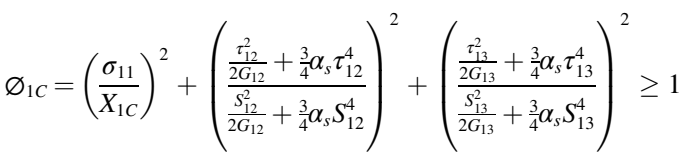

Matrix tensile cracking in direction 2 (SDV3), for $\sigma_{22} \quad 0$ :

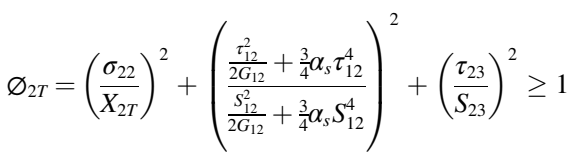

Matrix compressive cracking in direction 2 (SDV4), for $\sigma_{22}<0$ :

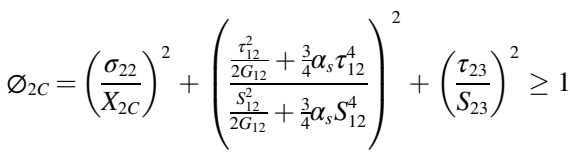

Matrix tensile cracking in direction 3 (SDV5), for $\sigma_{33} \quad 0$

$\varnothing_{3 T} \quad{\frac{\sigma_{33}}{X_{3 T}}}^{2}\left(\begin{array}{lll}\frac{\tau_{13}^{2}}{2 G_{13}} & \frac{3}{4} \alpha_{s} \tau_{13}^{4} \\ \frac{S_{13}^{2}}{2 G_{13}} & \frac{3}{4} \alpha_{s} S_{13}^{4}\end{array}\right)^{2} \quad \frac{\frac{\tau}{23}_{S_{23}}}{}{ }^{2} 1$

Matrix compressive cracking in direction 3 (SDV6), for $\sigma_{33}<0$ :

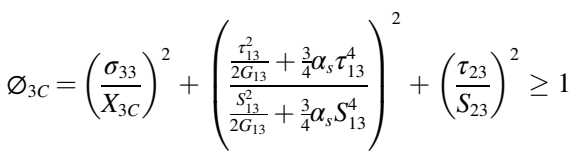

In Eqs. (7)-(12), $\sigma_{i j} i, j \quad 1,2,3$ are the stress components in the material coordinates of yarn. $X_{1 T}, X_{1 C}, X_{2 T}, X_{2 C}, X_{3 T}$ and $X_{3 C}$ are the tensile and compressive strengths of the yarns in each direction, $S_{12}, S_{13}$

Table 3

Mechanical properties of yarns and matrix.

\begin{tabular}{lllllll}
\hline & $E_{11} / \mathrm{GPa}$ & $E_{22} / \mathrm{GPa}$ & $G_{12} / \mathrm{GPa}$ & $G_{23} / \mathrm{GPa}$ & $v_{12}$ & $v_{23}$ \\
\hline Yarns & 59.1 & 22.8 & 8.7 & 9.0 & 0.25 & 0.27 \\
Matrix & 3.5 & & 1.3 & & 0.35 & \\
& $X_{t} / \mathrm{MPa}$ & $Y_{t} / \mathrm{MPa}$ & $X_{c} / \mathrm{MPa}$ & $Y_{c} / \mathrm{MPa}$ & $S_{12} / \mathrm{MPa}$ & $S_{23} / \mathrm{MPa}$ \\
Yarns & 2208 & 76 & 1600 & 164 & 61 & 41 \\
Matrix & 112 & & 241 & & 89.6 & \\
\hline
\end{tabular}

Table 4

Mechanical properties of interface.

\begin{tabular}{llllll}
\hline$E_{n n} / \mathrm{GPa}$ & $E_{s s} / \mathrm{GPa}$ & $E_{t t} / \mathrm{GPa}$ & $t_{n} / \mathrm{MPa}$ & $t_{s} / \mathrm{MPa}$ & $t_{t} / \mathrm{MPa}$ \\
\hline 3.0 & 1.15 & 1.15 & 49.5 & 43.5 & 43.5 \\
\hline
\end{tabular}

and $S_{23}$ are the shear strengths, respectively. $\alpha_{s}$ is the shear nonlinear factor; it was $2.4410{ }^{8} \mathrm{MPa}{ }^{3}$ in the simulations [26].

The material orientations of different yarns in the RVC are given in Fig. 7. For braiding and axial yarns, direction 2 was the y direction, i.e. the thickness direction of the RVC. Direction 2 of the radial yarns was the load axial direction, while their direction 3 was the width direction of the RVC.

The Von-Mises stress criterion was used to determine initial failure of the matrix (SDV7) since it was isotropic and its stress distribution was complex.

$\begin{array}{lllllllllll}\sigma_{1} & \sigma_{2}{ }^{2} & \sigma_{2} & \sigma_{3}{ }^{2} & \sigma_{3} & \sigma_{1}{ }^{2} & 6 & \tau_{12}^{2} & \tau_{23}^{2} & \tau_{31}^{2} & 2 \sigma_{m}^{2}\end{array}$

The process of property deterioration in the yarns and matrix played a significant role in the composite performance. After using the failure criterion to assess the onset of damage in the yarns or the matrix, a stiffness degradation model should be employed. In the simulations, the effect of damage on the material was characterized by the elastic constant at the integral point of the damage element. For different failure modes, in order to simulate effectively the anisotropic damage characteristics, the corresponding property degradation scheme should be developed to selectively reduce the respective material parameter. In this paper, a set of schemes of material-performance degradation was developed based on literatures [26,27], and the stiffness-degradation coefficients corresponding to respective failure mode are listed in Table 5.

The onset of damage at the interface is described by a quadratic damage criterion, which can be expressed as

$\left\{\frac{\left\langle t_{n}\right\rangle}{N}\right\}^{2} \quad\left\{\frac{t_{s}}{S}\right\}^{2} \quad\left\{\frac{t_{t}}{S}\right\}^{2} \quad 1$

Here, $N$ and $S$ are normal and shear strength of the interface, respectively. $\left\langle t_{n}\right\rangle$ is the Macaulay bracket equal to $\frac{t_{n}\left|t_{n}\right|}{2}$, since normal compressive stress has no contribution to the onset of damage. $t_{n}, t_{s}$ and $t_{t}$ are three stress components.

The damage-evolution process is based on the energy, analysis

$$
\frac{\left\langle G_{n}\right\rangle^{\alpha}}{G_{n}^{C}} \quad{\frac{G_{s}}{G_{s}^{C}}}^{\alpha} \quad{\frac{G_{t}}{G_{t}^{C}}}^{\alpha} 1
$$

where $\alpha$ is the exponent; its value is 1 in this paper. $G_{n}, G_{s}$ and $G_{t}$ are the

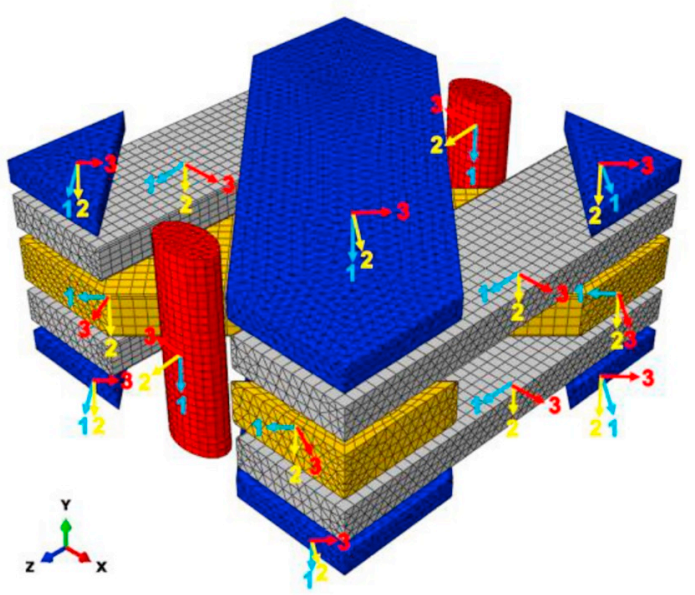

Fig. 7. Material orientation of different yarns. 
Table 5

Coefficients of material performance degradation.

\begin{tabular}{lcccccc}
\hline Failure mode & $E_{11}$ & $E_{22}$ & $E_{33}$ & $G_{12}$ & $G_{13}$ & $G_{23}$ \\
\hline $\begin{array}{l}\text { Fibre tensile failure } \\
\begin{array}{l}\text { Fibre compressive failure and } \\
\quad \text { fibre-matrix shear-out }\end{array}\end{array}$ & 0.01 & 0.01 & 0.01 & 0.01 & 0.01 & 0.01 \\
$\begin{array}{l}\text { Yarn-matrix tensile cracking in } \\
\text { direction 2 }\end{array}$ & 1.00 & 0.01 & 1.00 & 0.30 & 1.00 & 0.30 \\
$\begin{array}{l}\text { Yarn-matrix compressive cracking } \\
\quad \text { in direction 2 }\end{array}$ & 1.00 & 0.01 & 1.00 & 0.30 & 1.00 & 0.30 \\
$\begin{array}{l}\text { Yarn-matrix tensile cracking in } \\
\quad \text { direction 3 }\end{array}$ & 1.00 & 1.00 & 0.01 & 1.00 & 0.30 & 0.30 \\
$\begin{array}{l}\text { Yarn-matrix compressive cracking } \\
\text { in direction 3 }\end{array}$ & 1.00 & 1.00 & 0.01 & 1.00 & 0.30 & 0.30 \\
$\quad$ Matrix failure & 0.01 & 0.01 & 0.01 & 0.30 & 0.30 & 0.30 \\
\hline
\end{tabular}

energy in normal and shear direction caused by interface stress, respectively, while $G_{n}^{C}, G_{s}^{C}$ and $G_{t}^{C}$ are the respective critical fracture energy levels; they all are equal to $75 \mathrm{~J} / \mathrm{m}^{2}$ [20].

\section{Results and discussion}

\subsection{Comparison with experimental results}

The stress-strain relationships of the studied 3D multi-layer wrapping braided composite for tension, obtained from the experiments and with finite-element simulations, are shown in Fig. 8. The ultimate strain was not measured in the experiments. The stress-strain curve cased on the FEM result is between the highest and lowest experimental curves, indicating that the simulation results were in good agreement with the experimental data. The tensile elastic modulus obtained in simulations was $22.24 \mathrm{GPa}$; its difference from the experimental result was $2.4 \%$. The calculated Poisson's ratio was 0.35, close to the experimental results. The FEM curve reached the maximum value of $333 \mathrm{MPa}$ at about $1.99 \%$ strain, $4.1 \%$ higher than the average experimental strength.

It can be also seen in Fig. 8 that the FEM curve exhibited five main stages. The first stage is the initial stage, with a linear stress-strain relationship both in simulations and experiments. When the strain exceeded $0.50 \%$, the slope of the experimental curves decreased, indicating that stiffness began to diminish. However, the initial inflection point of the FEM results was at strain of about $0.60 \%$, i.e. somewhat later than in the experiments. When the strain was between $0.60 \%$ and $0.80 \%$, the slope of the FEM curve dropped significantly. After this stage, as the load increased, the stiffness continued decreasing. For the strain between $1.38 \%$ and $1.99 \%$, the slope of the FEM curve is almost constant, indicating that the main damage evolution stopped. At strain of about $1.99 \%$, the stress reached its peak of $333 \mathrm{MPa}$. Then, although strain continued to increase, the stress level decreased rapidly. It means that the material lost its load-bearing capacity and reflects the brittle character of failure.

\subsection{Mechanical response of unit cells}

Character of deformation of the studied braided composite under axial tensile load is shown in Fig. 9 for strain of $0.345 \%$ (deformation scale factor is 80). Apparently, all the boundary surfaces under such a load are warped, especially those perpendicular to the $\mathrm{Z}$ axis. A disparity in the character of warping for different surfaces was mainly caused by asymmetry of the underpinning geometric structure and mechanical properties. The surface with the negative $\mathrm{Z}$ normal deformed outwards, while that with the corresponding position on the surface with the positive $\mathrm{Z}$ normal deformed inwards to the same extent, and vice versa. All other corresponding boundary surfaces demonstrated similar behavior, ensuring the continuity of displacement thanks to the periodicity of unit cells.

Distributions of Von-Mises stresses of the whole model, as well as its

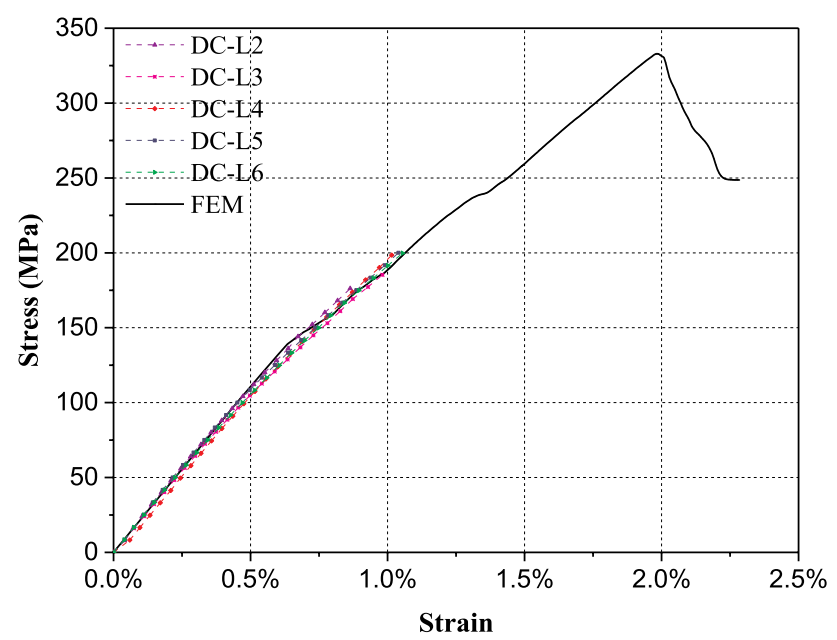

Fig. 8. Experimental and calculated stress-strain relationships of 3D multilayer wrapping braided composites under axial tensile load.

constituents - yarns, matrix and interface - at strain of $0.345 \%$ are shown in Fig. 10. The stress distributions of the whole model demonstrate the same stresses on the opposite boundary surfaces. Comparing stresses of each component, the average stress level in the yarns was much higher than that in the matrix and at the interface, indicating that the axial tensile load is mainly borne by the yarns. Stress concentration at the interfaces and contact areas between the yarns and the matrix can be clearly observed in Fig. 10b and c that, but it is practically no stress concentration in the yarns, and the stress distribution in each yarn is almost uniform. As shown in Fig. 10d, the stresses of axial yarns were about twice of those in the braiding yarns and ten times of those in the radial yarns. This demonstrates that the axial yarns bear the major load under axial tension, while the radial yarns hardly bear any axial load.

\subsection{Failure mechanisms}

The progressive damage process at the interface between the yarns and the matrix of the 3D braided composites under tensile load is presented in Fig. 11. The interface began to enter the plastic stage when the strain reached $0.35 \%$. After the strain continued to increase by $0.60 \%$, the interface between the radial yarns and the matrix reached the strength limit first and began breaking. Soon, one side of the interface was completely destroyed, resulting in the separation of the radial yarn and the matrix. Subsequently, one side of the other radial yarn separated from the matrix and the interfaces between the braiding yarns and the

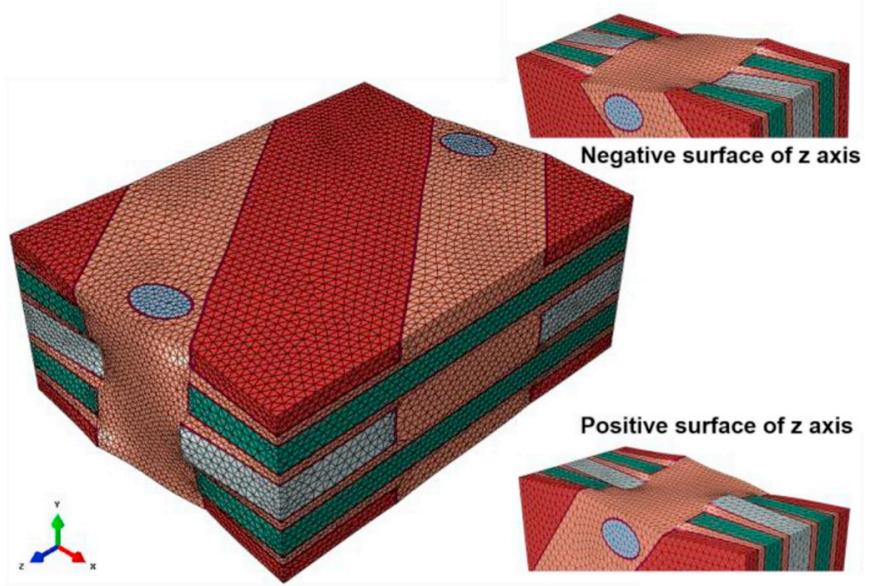

Fig. 9. Deformation character of RVC under axial tension. 

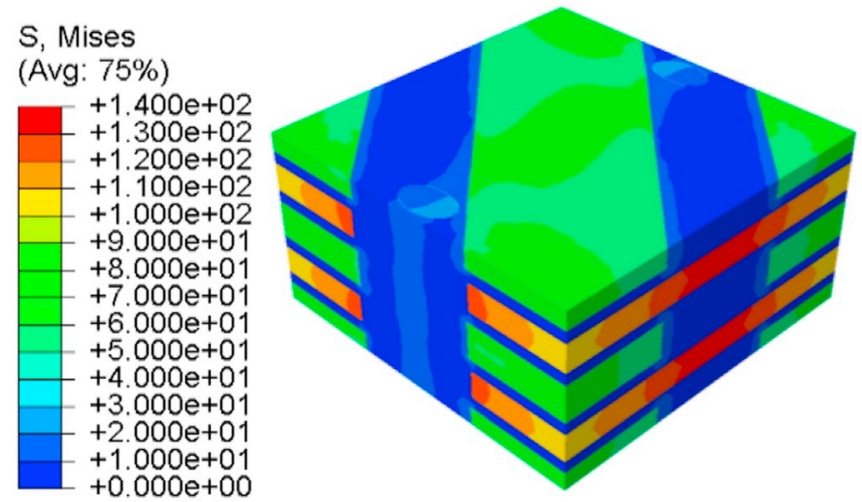

(a)

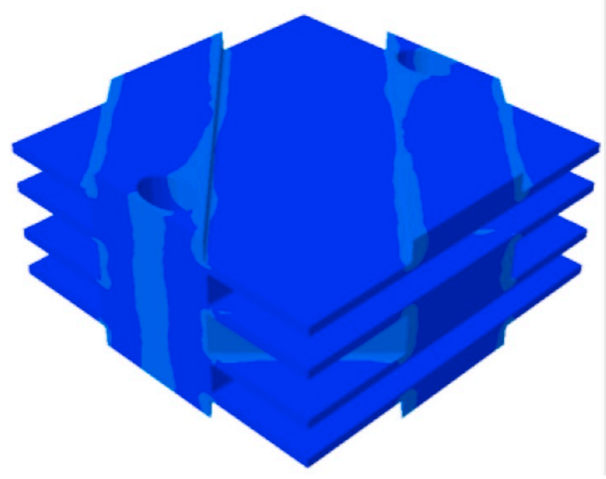

(c)

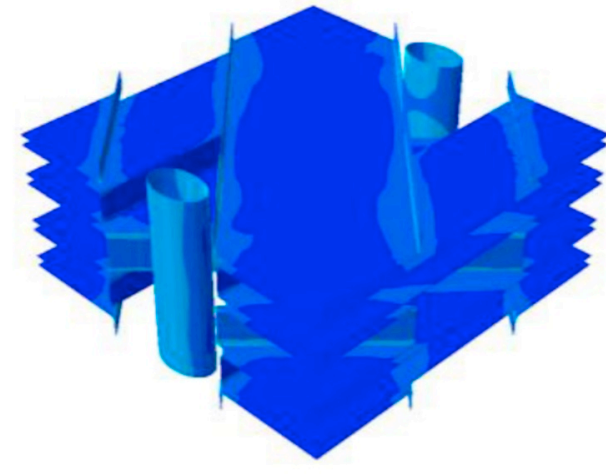

(b)

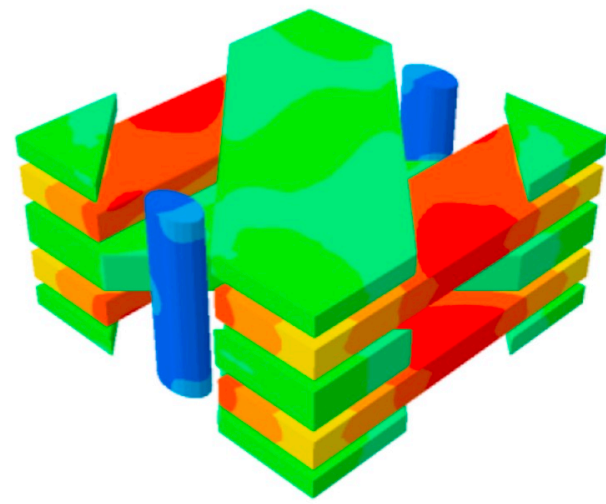

(d)

Fig. 10. Distributions of Von-Mises stress in unit cell at strain of $0.345 \%$ : (a) whole model; (b) interface; (c) matrix; (d) yarns.

matrix began to damage. The damage continued to increase until the strain reached its ultimate strain value, at which point two sides of each braiding yarn almost separated from the matrix. As the strain increased, large areas of the interface between the axial yarns and the matrix entered the plastic stage of deformation, and it can be predicted that the interface separation should occur soon.

The progressive damage processes of different failure modes in the studied composite under tensile load are shown in Fig. 12. The processes of fibre compressive failure and fibre-matrix shear-out, yarn-matrix compressive cracking in direction 2 and yarn-matrix compressive cracking in direction 3 are not shown since they were rarely insignificant. The red colour in Fig. 12 denotes damaged areas and the blue colour the undamaged ones.

The evolution of fibre tensile failure is presented in Fig. 12a. At strain

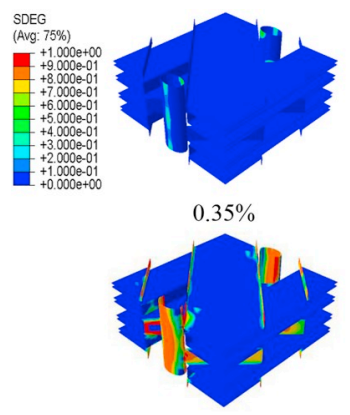

$1.37 \%$

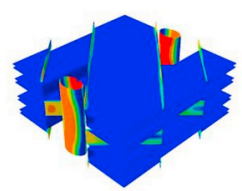

$0.95 \%$

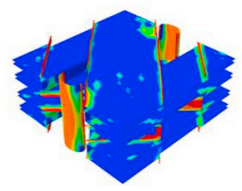

$1.99 \%$

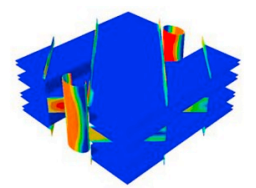

$0.98 \%$

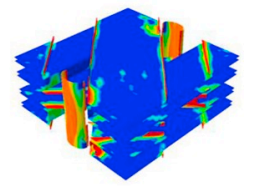

$2.16 \%$
Fig. 11. Progressive damage process in interface under tensile load. of $0.69 \%$, the initial damage first appeared in the braiding yarns, gradually expanding into them with the increase of tension. The fibre tensile failure began to occur in axial yarns at strain of $1.96 \%$. Damage in these yarns extended quickly along the axial direction; its expansion was faster than that in the braiding yarns. After additional stretching by $0.03 \%$, the model reaches the strength limit and lost its load-bearing capacity. During the entire process, there was no fibre tensile failure in the radial yarns.

Evolution of yarn-matrix tensile cracking in directions 2 and 3 are shown in Fig. 12b and c. The yarn-matrix tensile cracking occurred first in the radial yarns, then in the braiding yarns, and, finally, in the axial yarns. The damage propagation in the width direction was faster than that in the thickness direction. At strain of $1.30 \%$, almost all areas of the braiding and radial yarns suffered from yarn-matrix tensile cracking in the width direction. When the strain reached 1.99\% (Fig. 12c), the respective damage zones along the axial direction were clearly observed on the surfaces of the axial yarns, meaning that they were divided into several parts in the width direction.

The process of matrix-failure evolution is presented in Fig. 12d. The initial damage of the matrix occurred later than that in the yarns. It first appeared in the areas near the radial yarns; the stress-concentration areas were analyzed in Section 5.2. With the increasing tensile load, the damage expanded along the interface, and the damage area gradually coalesced.

The diagrams with damage evolution show only the process on the surfaces of the yarns and the matrix. In order to assess the internal damage evolution, the volume of damage caused by different failure modes was calculated; the relationships between the failure volume fraction and strain are given in Fig. 13. The failure volume fraction represents the ratio of the damage volume to the total volume. The 
(a)
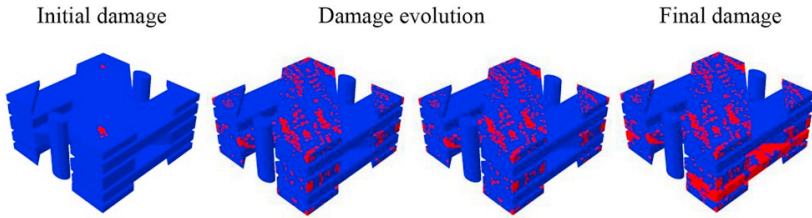

(b)
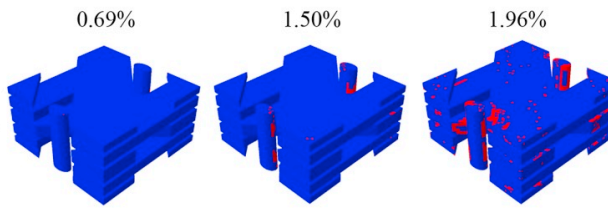

$1.99 \%$

(c)

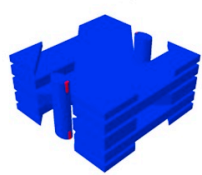

$0.69 \%$

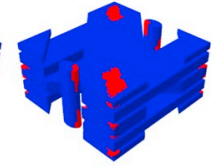

$1.32 \%$
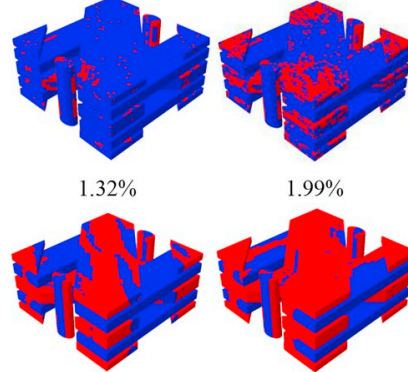

(d)

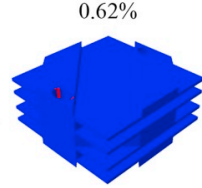

$0.97 \%$

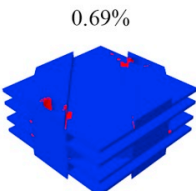

$1.44 \%$

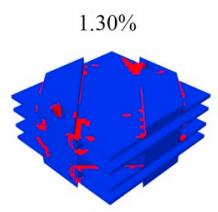

$1.86 \%$

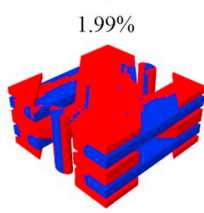

$99 \%$

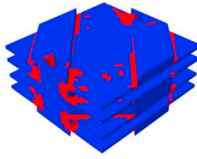

$1.99 \%$

Fig. 12. Progressive damage processes in yarns and matrix under tensile load: (a) fibre tensile failure; (b) yarn-matrix tensile cracking in direction 2; (c) yarnmatrix tensile cracking in direction 3; (d) matrix failure.

failure volume fraction of different failure modes in the whole model are shown in Fig. 13a, while Fig. 13b-d show the failure volume fraction of the same failure mode in different types of yarns.

No failure mode was found until the strain reached $0.60 \%$, which corresponded to the linear segment of the FEM curve in Fig. 7. When the strain exceeded $0.60 \%$, the yarn-matrix tensile cracking in direction 3

first occurred gradually inducing other types of yarn damage. The failure volume fraction of this mode increased significantly, leading to an obvious drop of the slope of the stress-strain curve. Compared with other failure modes, the failure volume fraction of this mode was the largest and its rise was fastest. As seen in Fig. 13d, when the strain was between $0.62 \%$ and $1.00 \%$, there were two jumps of damage in both braiding and radial yarns corresponding to Fig. 13a. No damage could be found in the axial yarns, indicating that the damage development only occurred in the braiding and radial yarns during this stage. When the strain was between $1.00 \%$ and $1.50 \%$, the damage in the radial yarns tends to be gradual, while damage in the braiding yarns expanded steadily. The damage in the axial yarns was not discovered until strain of $1.20 \%$. When the strain was between $1.50 \%$ and $1.99 \%$, the damage accumulation mainly occurred in the axial yarns, while the failure volume for the braiding yarns tended to the limit, and damage propagation in the radial yarns almost stopped. All these factors led to the decrease in the grown of the yarn-matrix tensile cracking in direction 3 (Fig. 13a).

The failure volume fractions of fibre tensile failure and yarn-matrix tensile cracking in direction 2 were approximately the same at strain between $0.62 \%$ and $1.40 \%$, but for different reasons. From Fig. $13 \mathrm{~b}$ and $c$, it is clear that fibre tensile failure only occurred in the braiding yarns, while yarn-matrix tensile cracking in direction 2 occurred in both braiding and radial yarns. Although there were several stages of swift jumps for fibre tensile failure in the braiding yarns, the failure volume was still small. After that, the failure volume fraction of fibre tensile failure was stable, while that of yarn-matrix tensile cracking in direction 2 increased steadily. Damage extension in the radial yarns almost stopped when the strain was higher than $1.40 \%$ (Fig. 13c), but the speed of yarn-matrix tensile cracking in direction 2 did not decline. This was because the volume of the radial yarns was only one-tenth of that in the braiding yarns. For strain higher than 1.90\%, the extension of fibre tensile failure and yarn-matrix tensile cracking in the thickness direction mainly happened in the axial yarns, and its speed was much higher than that for the braiding yarns.

At strain of $0.97 \%$, the matrix began to fail and the expansion speed

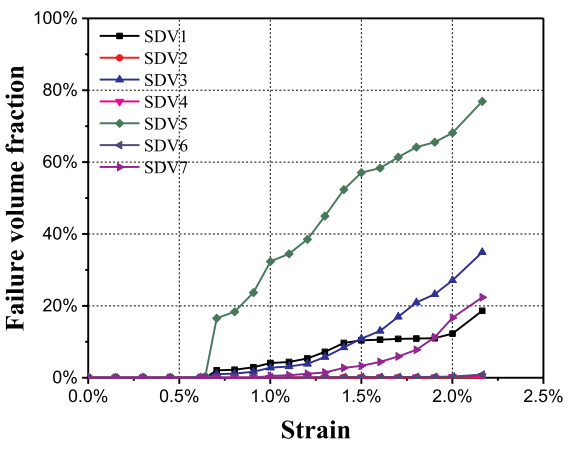

(a)

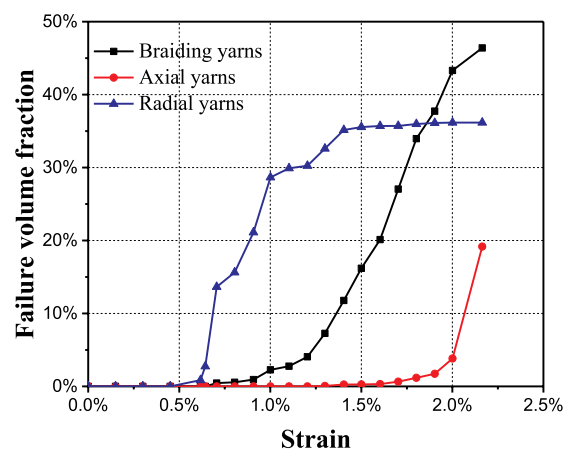

(c)

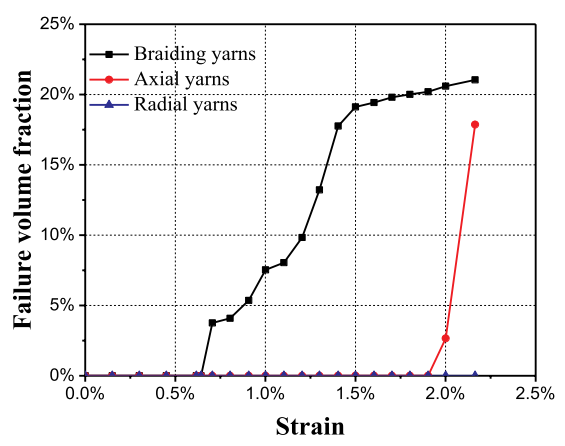

(b)

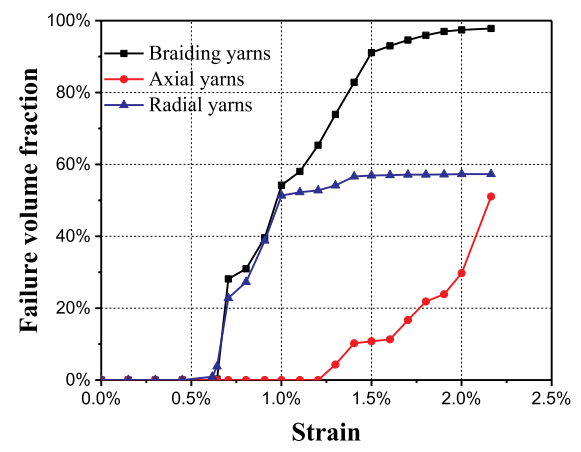

(d)
Fig. 13. Evolution of failure volume fraction with strain: (a) different failure modes (SDV1 - fibre tensile failure; SDV2 - fibre compressive failure and fibre-matrix shear-out; SDV3 - yarn-matrix tensile cracking in direction 2; SDV4 - yarn-matrix compressive cracking in direction 2; SDV5 - yarnmatrix tensile cracking in direction 3; SDV6 - yarnmatrix compressive cracking in direction 3; SDV7 matrix failure); (b) fibre tensile failure; (c) yarnmatrix tensile cracking in direction 2; (d) yarnmatrix tensile cracking in direction 3. 
increased with the load increase. The fibre compressive failure, fibrematrix shear-out and yarn-matrix compressive cracking did not occur until the strain of $1.86 \%$, and the respective failure volume fractions were very small.

The stress-strain curve reached the peak value at strain of $1.99 \%$. At this point, the yarn-matrix tensile cracking in the width direction was the main damage mode, and its failure volume fraction was $68.0 \%$, while that of fibre tensile failure and yarn-matrix tensile cracking in the thickness direction were $11.8 \%$ and $27.8 \%$, respectively. The failure volume fraction of the matrix also reached $16.7 \%$. When the strain exceeded the limit value, various failure modes expanded rapidly in the axial yarns, resulting in a decrease of the stress-strain curve in Fig. 7.

\section{Conclusion}

In this study, mechanical properties and failure mechanisms of the 3D multi-layer wrapping braided composite under tensile load were investigated in experiments and simulations. In the simulation, the geometric model and the respective FEM model were based on the braiding rule and observation of the composite. The interface elements between the yarns and the matrix were applied to improve the accuracy of simulations. The 3D Hashin criteria and Von-Mises stress criterion were employed as damage initiation criteria of the yarns and the matrix, respectively. By comparing the simulation results with the test data, the following conclusions can be made:

The tensile elastic modulus and strength obtained in numerical simulations were both in a good agreement with the experimental results. The simulated Poisson's ratio was close to the experimental level and the error of the elastic modulus between the numerical and experimental results was $2.4 \%$. The predicted tensile strength was $4.1 \%$ higher than the average experimental strength.

When the 3D multi-layer wrapping braided composites was subjected to the axial tensile load, the axial yarns were its main load-bearing constituent and the radial yarns are only a small portion of the load. The composite material lost its load-bearing capacity when fibres in the axial yarns began to break.

Under the axial tensile load, the main failure mode of the yarns was the yarn-matrix tensile cracking in the width direction, followed by the yarn-matrix tensile cracking in the thickness direction and fibre tensile failure. Furthermore, the yarn-matrix compressive cracking, fibre compressive failure and fibre-matrix shear-out were almost absent.

\section{Acknowledgements}

This work was supported by the China Scholarship Council (Grant No. 201706830061), the Fundamental Research Funds for the Central Universities (Grant No. NS2019001), Shanghai Aerospace Science and Technology Innovation Fund (Grant No. SAST2018-071) and the Priority Academic Program Development of Jiangsu Higher Education Institutions.

\section{References}

[1] Mouritz AP, Bannister MK, Falzon PJ, et al. Review of applications for advanced three-dimensional fibre textile composites. Compos Appl Sci Manuf 1999;30(12): 1445-61.

[2] Ayranci C, Carey J. 2D braided composites: a review for stiffness critical applications. Compos Struct 2008;85(1):43-58.

[3] Bilisik K, Bilisik K. Three-dimensional braiding for composites: a review. Text Res J 2013;83(13):1414-36.

[4] Wang C, Roy A, Silberschmidt VV, et al. Modelling of damage evolution in braided composites: recent developments. Mech Adv Mater Mod Processes 2017;3(1):15.

[5] Kalidindi SR, Abusafieh A. Longitudinal and transverse moduli and strengths of low angle 3D braided composites. J Compos Mater 1996;30(8):885-905.

[6] Sankar BV, Marrey RV. Analytical method for micromechanics of textile composites. Compos Sci Technol 1997;57(6):703-13.

[7] Cox BN, Carter WC, Fleck NA. A binary model of textile composites-I. Formulation. Acta Metall Mater 1994;42(10):3463-79.

[8] Xu J, Cox BN, Mcglockton MA, et al. A binary model of textile composites-II. The elastic regime. Acta Metall Mater 1995;43(9):3511-24.

[9] Cui JZ, Shih TM, Wang YL. Two-scale analysis method for bodies with small periodic configurations. Struct Eng Mech 1999;7(6):601-14.

[10] Tang ZX, Postle R. Mechanics of three-dimensional braided structures for composite materials - Part II: prediction of the elastic moduli. Compos Struct 2001; 51(4):451-7.

[11] Chen L, Tao XM, Choy CL. Mechanical analysis of 3D braided composites by the finite multiphase element method. Compos Sci Technol 1999;59(16):2383-91.

[12] Sun J, Zhou G, Zhou C. Microstructure and mechanical properties of 3D surfacecore 4-directional braided composites. J Mater Sci 2015;50(22):7398-412.

[13] Li DS, Fang DN, Jiang N, et al. Finite element modeling of mechanical properties of 3D five-directional rectangular braided composites. Compos B Eng 2011;42(6): 1373-85.

[14] Sun HY, Qiao X. Prediction of the mechanical properties of three-dimensionally braided composites. Compos Sci Technol 1997;57(6):623-9.

[15] Zuo WW, Xiao LY, Liao DX. Statistical strength analyses of the 3D braided composites. Compos Sci Technol 2007;67(10):2095-102.

[16] Yu XG, Cui JZ. The prediction on mechanical properties of 4-step braided composites via two-scale method. Compos Sci Technol 2007;67(3-4):471-80.

[17] Li DS, Lu ZX, Chen L, et al. Microstructure and mechanical properties of threedimensional five-directional braided composites. Int J Solids Struct 2009;46(18): 3422-32.

[18] Jiang L, Zeng T, Yan S, et al. Theoretical prediction on the mechanical properties of 3D braided composites using a helix geometry model. Compos Struct 2013;100(5): $511-6$.

[19] Wang C, Zhong Y, Adaikalaraj PFB, et al. Strength prediction for bi-axial braided composites by a multi-scale modelling approach. J Mater Sci 2016;51(12): 6002-18.

[20] Fang G, Liang J, Wang B, et al. Effect of interface properties on mechanical behavior of 3D four-directional braided composites with large braid angle subjected to uniaxial tension. Appl Compos Mater 2011;18(5):449-65.

[21] Deng J, Zhou G, Ji L, et al. Mechanical behaviour of 3D multi-layer braided composites: experimental, numerical and theoretical study. Appl Compos Mater 2017;24(6):1509-23.

[22] Xia Z, Zhang Y, Ellyin F. A unified periodical boundary conditions for representative volume elements of composites and applications. Int J Solids Struct 2003;40(8):1907-21.

[23] Chamis CC. Mechanics of composite materials - past, present and future. J Compos Technol Res 1989;11(1):3-14.

[24] Hashin Z. Failure criteria for unidirectional fiber composites. J Appl Mech 1980;47 (2):329-34.

[25] Zhou Y, Lu Z, Yang Z. Progressive damage analysis and strength prediction of 2D plain weave composites. Compos B Eng 2013;47(3):220-9.

[26] Sun J. Research on mechanical properties and progressive damage of 3D surfacecore braided composites (Thesis). Nanjing University of Aeronautics and Astronautics; 2016 [In chinese)].

[27] Blackketter DM, Walrath DE, Hansen AC. Modeling damage in a plain weave fabric-reinforced composite material. J Compos Technol Res 1993;15(2):136-42. 